\title{
Tumor Growth Parameters Estimation and Source Localization From a Unique Time Point: Application to Low-grade Gliomas
}

\author{
Islem Rekik ${ }^{\mathrm{a}}$, Stéphanie Allassonnière ${ }^{\mathrm{b}}$, Olivier Clatz ${ }^{\mathrm{a}}$, Ezequiel Geremia ${ }^{\mathrm{a}}$, \\ Erin Stretton ${ }^{\mathrm{a}}$, Hervé Delingette ${ }^{\mathrm{a}}$, Nicholas Ayache $^{\mathrm{a}}$ \\ ${ }^{a}$ INRIA, Asclepios Project, 2004 Route des Lucioles, BP 93, F-06902 Sophia Antipolis \\ Cedex, France \\ ${ }^{b}$ CMAP Ecole Polytechnique, Route de Saclay, 91128 Palaiseau France
}

\begin{abstract}
Coupling time series of MR Images with reaction-diffusion-based models has provided interesting ways to better understand the proliferative-invasive aspect of glial cells in tumors. In this paper, we address a different formulation of the inverse problem: from a single time point image of a non-swollen brain tumor, estimate the tumor source location and the diffusivity ratio between white and grey matter, while exploring the possibility to predict the further extent of the observed tumor at later time points in low-grade gliomas. The synthetic and clinical results show the stability of the located source and its varying distance from the tumor barycenter and how the estimated ratio controls the spikiness of the tumor.
\end{abstract}

Keywords: diffusivity ratio, source estimation, Eikonal Equation, reaction-diffusion glioma growth modeling

\section{Introduction}

Brain gliomas represent about $50 \%$ of all primary brain tumors [1] and can be classified according to their grade of malignancy. Low grade gliomas

Email addresses: islem.rekik@gmail.com (Islem Rekik), stephanie.allassonniere@polytechnique.edu (Stéphanie Allassonnière), olivier.Clatz@inria.fr (Olivier Clatz), ezequiel.Geremia@inria.fr (Ezequiel Geremia), erin.stretton@gmail.com (Erin Stretton), herve.Delingette@inria.fr (Hervé Delingette), Nicholas.Ayache@inria.fr (Nicholas Ayache) 
(LGG) are slow invaders of brain tissue as they keep growing for many years, presenting one of the most controversial decision treatment areas. High grade gliomas (HGG) remain unfortunately incurable with an average life expectancy of one year after its discovery, eventually creating symptoms due to an increase of the intracranial pressure or swelling around the tumor. The diagnosis of brain gliomas includes the analysis of various MRI sequences of the brain which partially reveal the tumor invasion. Based on those images and other clinical information, neurologists try to determine the grade of the gliomas and to estimate their current and further spatial extent and if possible their source location.

For more than a decade, mathematical models of brain tumors have been devised to help clinicians answer these questions. Microscopic models study the cellular mechanisms $[2,3,4,5]$ that explain the growth dynamics of gliomas at a microscopic scale. On the other hand, macroscopic models pioneered by Murray [6] describe the evolution of tumor cell density. However, those quantities cannot be directly observed in clinical medical images, but it is commonly assumed that visible tumor boundaries correspond to an isovalue of this density. More realistic reaction-diffusion models have been proposed by Swanson et al. [7] based on the fact that tumor cells migrate faster on white matter fibre tracts myelin sheaths [8]. They have been further refined by Jbabdi et al. [9] and Clatz et al. [10] by considering an anisotropic diffusion in the white matter whose diffusion tensor is estimated from those acquired in DT-MRI.

A key issue for those models to answer clinical questions is their personalization, i.e. the estimation of some patient-specific parameters from medical images. The main parameters to be identified based on reaction-diffusion models were pointed out in [11] as a combination of tumor diffusion tensors in white and grey matter, its proliferation rate, its initial point and its initial time. Several authors have estimated patient-specific parameters manually [10] or through major model simplifications [12]. Colin et al. in [13] used a reduced model based on Proper Orthogonal Decomposition (POD) in order to identify growth parameters of pulmonary nodules in CT images. Konukoglu et al. $[14,15,16]$ have proposed an approach to automatically and accurately personalize brain tumor models. They first remarked that given time series of brain MR images, only the motion of a tumor front can be observed and therefore only the three following parameters can be recovered : diffusivity in the white $d_{w}$ and grey matter $d_{g}$ as well as the initial time $T_{0}$. Furthermore, since the tumor cell density are only observed in MR images through visible 
tumor boundaries corresponding to an isovalue, the reaction-diffusion equations can be advantageously replaced by an Anisotropic Eikonal Equation (AEE) [16] which models the time at which a tumor front reaches a given point. By minimizing the distance between the segmented tumor and the simulated one, they were able to estimate uniquely those three parameters and test the prediction of future tumor evolution from at least a pair of images.

In this paper, we tackle a slightly different problem than the one approached by previous authors. Instead of estimating the speed of tumor growth from a time series of images, we aim at characterizing the nature of the glioma, more precisely LGG, from a single MR image. Indeed, we hypothesized that the tumor shape is dependent on the proliferating or infiltrating nature of the tumor. Contrary to HGG where the presence of brain edema is common and usually associated with tumor malignancy, LGG are slowly growing tumors with a minimal surrounding edema $[17,18,19]$. Since our methodology main focus is on LGG, we will not consider the edema-induced mass effect in our further formulation of the tumor growth model. Therefore, the anatomical boundaries such as the ventricles' will remain static as the tumor grows.

Given a segmented brain glioma from an MR image, we solve an inverse problem in order to estimate the diffusivity ratio $d_{w} / d_{g}$ and the tumor source position. By localizing the tumor source and estimating the invaded tissue characteristic using this ratio $d_{w} / d_{g}$ our objective is to provide clinicians with new indices that can be used for diagnosis from the first acquired MR images, combined with a subsequent prediction of tumor invasive margins as it grows from the initially observed boundary. This additional information may help in surgical and/or radio-therapeutic treatment planning especially when it comes to determining the margins for applying the therapy. The problem of tumor seed localization was recently raised in [20], where a reaction-diffusion based joint estimation of tumor evolution parameters was combined with a multimodal deformable registration framework. This approach focuses on the MR image registration with an atlas providing the estimation of the initial seed location as a by-product. Later on, it was extended to a joint segmentation and deformable registration between multi-sequence brain MR image with gliomas and a probabilistic brain atlas of a healthy population [21, 22 . These recent publications targeting the localization of tumor seeds, the quantification of the mass effect and its white matter diffusion coefficient was based on a normal atlas. 
In this paper, we investigate a different problem from registration or segmentation in low-grade gliomas. Our approach addresses a spatio-temporal tumor evolution with the estimation of the diffusivity ratio and the position of its source. Additionally, after solving the inverse problem, we evaluate the stability of the location of tumor sources over time and analyze the relationship between the tumor source and tumor barycenter as both locations have been assumed to match in past studies [23, 24, 25, 26, 27]. Finally, knowing the diffusivity ratio $d_{w} / d_{g}$ and the tumor source from a single image, we evaluate whether this information gives insights into predicting further tumor shape evolution in two distinct cases of low-grade gliomas.

\section{Material and Method}

\subsection{MR Glioma images}

In this work, we assume that for a given patient, one MR FLAIR image of the brain has been acquired, showing visible boundaries of glioma cells. We also assume that a Diffusion Tensor MR Image (DT-MRI) is also available and acquired at the same time as the FLAIR image. While FLAIR images are acquired in routine on patients with brain tumors, this is unfortunately not the case for DT-MRI. The extent of the tumor has been manually segmented in FLAIR images. Similarly, brain masks have been manually delineated on those images from which brain ventricles have been removed by a simple thresholding of the signal. Also white matter regions have been isolated by thresholding the voxels with the largest eigenvalue in the DT-MRI.

Objective: From the segmented tumor in the FLAIR image, our objective is to provide a quantitative analysis of the tumor shape which is not simply based on geometry (spheroid vs star-shaped) but based on simple biophysics growth principles. Indeed, two quantities are estimated in this analysis: the tumor source position and the diffusivity ratio between white matter and grey matter. This information may provide additional hints about the nature and the future progression of the tumor.

Data issues: Collecting LGG data with DT-MRIs is not a very straightforward task since diffusion MR is a quite recent technology [28], rarely used in the common clinical practice and furthermore acquired DT-MRI may have various anomalies like holes, low resolution and signal distortion. This is particularly true around any tumor lesion. This lack of information may be compensated by assuming the symmetry of the brain. For this, we perform a symmetrization process to "reinitialize" the region where the tumor grew 
and induced a diffusion signal distortion. Hence, for a proper simulation of tumor growth, we have corrected the tensor field by making the hypothesis that the DT MRI was originally symmetric with respect to the mid-sagittal plane. Thus the DT-MRI voxels corresponding to the largest extent of the tumor have been modified by symmetrizing and copying the voxels from the healthy brain hemisphere. However, this symmetrization process is prone to the following difficulties:

a- the DT MRI is asymmetric especially in white matter where about $50 \%$ of the contralateral tumor volume has zero Fractional Anisotropy (FA) values, while in the corresponding affected region where the tumor grew, the diffusion signal exists with a remarkable distortion (see Fig 1-a where the FA tumor map is darker than the contralateral part with a significant absence of symmetry).

b- even with a reliable DT-MRI symmetry, about $50 \%$ of the contralateral non-pathological symmetric volume to the tumor volume has no DT (i.e FA) signal. In Fig 2-a, the presence of large black hole in the FA signal in the contralateral part to the tumor invasion area presents a major barrier to diffusion tensor-guided glioma evolution simulation. To cope with this DT signal-missing problem, an interpolation algorithm based on isotropic diffusion (solving the heat equation) was applied to estimate the missing tensors from neighboring regions. This is done by applying a Gaussian convolution separately on the six components of the diffusion tensors.

Dataset: By excluding LGG cases with completely distorted or missing DTI, we succeeded to include four LGG patients. The first case, a.k.a patient A, has developed a second grade astrocytoma classified as a low-grade glioma. Four successive time points of T2 flair MR images with a resolution of $0.99 \times 1 \times 2.16 \mathrm{~mm}^{3}$ were acquired and visible tumor boundaries were manually delineated by an expert. A DT-MRI image was also acquired at the first acquisition time point. As expected, the white matter fiber tracts are perturbed by the tumor growth and the DT-MRI signal near the tumor does not capture the original diffusion tensors of the brain at the onset of the disease. Therefore, we used the symmetrization process to reset the affected area to non-pathologically spatially deviated tensors (see Fig 3 where the white matter diffusion tracts were beautifully recovered).

The second case, a.k.a patient B, suffers from a low-grade glioma and four MR images were acquired at distinct time points with a resolution of $0.89 \times 0.97 \times 0.97 \mathrm{~mm}^{3}$. Only one DT-MRI was acquired during the initial scanning process and the tumor region of the DT-MRI has been symmetrized 
similarly to patient A. In addition, the DT-MRI includes small holes in the opposite region to the tumor that were successfully interpolated.

The third case, a.k.a patient $\mathrm{C}$, with LGG has three acquisition time points with a resolution of $2 \times 2 \times 2 \mathrm{~mm}^{3}$. In this particular case, displayed in Fig 1, we encountered difficulty (-a-) where the DT-MRI is not fully symmetric.

The fourth case, a.k.a patient D, with a resolution of $2 \times 2 \times 2 \mathrm{~mm}^{3}$ was not symmetrized due to the large holes in the symmetric region to the tumor as show in Fig 2-a. Therefore, we interpolated the holes in the affected tissue without the symmetrization process.

\subsection{Tumor Growth Modeling : from reaction-diffusion to Eikonal equations}

Glial cells dynamics are essentially governed by two biological phenomena : proliferation and invasion. They can be jointly modeled by a reactiondiffusion equation which describes the change over time of the normalized tumor cell density $u$ :

$$
\left\{\begin{array}{l}
\frac{\partial u}{\partial t}=\nabla \cdot(\mathbf{D}(\mathbf{x}) \nabla u)+\rho u(1-u) \\
\mathbf{D} \nabla u \cdot n_{\partial \Omega}=0
\end{array}\right.
$$

where $\rho$ is the proliferation rate, $\mathbf{D}$ the local diffusion tensor, and $n_{\partial \Omega}$ is the normal vector at the domain boundary surface $\partial \Omega$. In the first equation, the proliferation of tumor cells follows a logistic growth parameterized by $\rho$ whereas the tumor infiltration into neighboring neural fibers is captured by an anisotropic diffusion parameterized by $\mathbf{D}$. The second equation indicates that there is no flux of tumor cells outside the domain $\Omega$.

The diffusion tensor is a definite positive and symmetric $3 \times 3$ matrix whose value may be linked to Diffusion Tensor MRI (DT-MRI) [9]. Indeed, it characterizes the motility of tumor cells that is considered to be isotropic in grey matter but anisotropic in white matter. More precisely, the tumor diffusion tensor (TDT) may be written as $\mathbf{D}(\mathbf{x})=d_{g} \mathbf{I}_{3}$ in grey matter, where $d_{g}$ is the diffusivity coefficient.

In white matter, there are several approaches to link the TDT $\mathbf{D}(\mathbf{x})$ with the DT-MRI signal $\mathbf{D}_{\text {water }}(\mathbf{x})$. Clatz et al. [10] proposed to have $\mathbf{D}(\mathbf{x})$ proportional to $\mathbf{D}_{\mathrm{water}}(\mathbf{x})$ whereas Jbabdi et al. [9] have introduced a formulation which takes into account the possible equality of the two largest eigenvalues corresponding to a possible fiber crossing. Due to high anisotropies of $\mathbf{D}_{\text {water }}(\mathbf{x})$ in most parts of the white matter, these two approaches lead 
however to diffusivities that are much lower than $d_{g}$ in the directions orthogonal to the fibers, which is questionable. Furthermore, the high ratios of anisotropy encountered in those two expressions also lead to large computational times.

In this paper, we propose to use the following white matter tumor diffusion tensor:

$$
\mathbf{D}(\mathbf{x})=\mathbf{V}(\mathbf{x})\left[\operatorname{diag}\left(e_{1}(\mathbf{x}) d_{w}, d_{g}, d_{g}\right)\right] \mathbf{V}(\mathbf{x})^{T}
$$

where $d_{w}$ is the white matter diffusivity coefficient, $\mathbf{V}(\mathbf{x})$ represents the matrix of sorted eigenvectors of $\mathbf{D}_{\mathrm{water}}(\mathbf{x})$ and $e_{1}(\mathbf{x})$, is the normalized largest eigenvalue (between 0 and 1 ) of $\mathbf{D}_{\text {water }}(\mathbf{x})$. With this choice, tensors have a non-homogeneous anisotropy ratio which is always less than $d_{w} / d_{g}$ but is maximized at the center of the white matter fibers and continuously decreases towards their boundaries. By simply dividing the duration of tumor evolution simulation of its propagating front using our adopted diffusion tensor 2 by the simulation duration as we used the diffusion tensor formulas presented in [9] (we precisely refer the reader to formulas A11 and A12), we have noticed that our choice speeds-up the computational time by a factor of 200 without any significant differences in performance. In fact, the use of a more nonlinear (more anisotropic) diffusion tensor field increases the computational time of the solution as the characteristic direction of the recursive anisotropic fast marching algorithm used to solve the AEE 5 becomes harder to find. This also may be explained by the fact that below a certain anisotropy ratio, the difference in tumor growth simulation is hardly noticeable.

The reaction-diffusion equation (1) is not practical when dealing with clinical images. Indeed, in MR images tumor cell density $u$ cannot be observed but only the visible tumor boundary can. Hence, a front motion approximation for the reaction-diffusion equation was introduced by Konukoglu et al. [16] assuming that the visible contour is associated with iso-density contour $u=0.4$ [29]. They introduced an Anisotropic Eikonal Equation (AEE) describing the time $T(\mathbf{x})$ at which the evolving tumor front passes through the location $\mathbf{x}$. In its simplest form, the AEE writes as:

$$
F \sqrt{\nabla T^{T} \mathbf{D} \nabla T}=1 \text { with } F=2 \sqrt{\rho}
$$

However, such approximation of equation 1 is too simple and Konukoglu et al. then proposed to account for the fact that the tumor front speed increases over time to reach an asymptotic value equal to $2 \sqrt{\rho \mathbf{n}^{T} \mathbf{D n}}$ where $\mathbf{n}$ is the normal direction of the front. The time-dependent speed of the 
propagating tumor front was introduced in [16] as:

$$
F=\frac{4 \rho T-3}{2 \sqrt{\rho} T}
$$

Furthermore, the front curvature $\kappa_{\text {eff }}(\mathbf{x})$ also plays a role in the front speed as the front slows at high curvature points. This is especially important at the early stage of the tumor growth when the front is similar to a small sphere. With this additional hypothesis, the speed term becomes :

$$
F=\frac{4 \rho T-3}{2 \sqrt{\rho} T}-0.3 \sqrt{\rho}\left(1-e^{-\left|\kappa_{e f f}\right| / 0.3 \sqrt{\rho}}\right)
$$

This last formulation is no longer a Hamilton-Jacobi equation due to the second-order curvature term and therefore cannot be solved by fast sweeping methods such as the Anisotropic Fast Marching (AFM) [14]. However, a multi-pass approach was proposed [16] to solve efficiently this equation by applying several times the AFM method while estimating the curvature front from previous iterations. The AFM method is recursive and the larger the tensor anisotropy the more iterations are needed to compute the characteristic direction of equation (5). Our white matter TDT of equation (2) limits the anisotropy ratio and therefore leads to reasonable computational times (typically few minutes for a tumor growth from a seed point).

\subsection{Parameter estimation problem from a unique $M R$ image}

Based on the previously exposed mathematical model, we can simulate the growth of a glioma given its initial source $S(\mathbf{x})$ for which we assume that $T(S(\mathbf{x}))=0$. From this boundary condition and the knowledge of diffusivity $d_{w}, d_{g}$ and proliferation rate $\rho$ we can compute the time $T(\mathbf{x})$ at which the visible tumor front reaches a given point. The isocontours of the field $T(\mathbf{x})$ correspond to the successive shapes of the visible tumor boundary over time as shown in Fig 4. The speed on the front is not constant but its asymptotic value is $2 \sqrt{\rho d_{g}}$ in grey matter and $2 \sqrt{\rho d_{w}}$ in white matter.

In this paper, we are interested in solving the following inverse problem: given a visible tumor boundary $S_{\text {Seg }}$ in an MR image, can we extract the growth parameters $d_{w}, d_{g}, \rho, T_{\mathrm{Obs}}$ and source location $S(\mathbf{x})$ that best explain the observed tumor boundary? The duration $T_{\mathrm{Obs}}$ between the onset of the tumor and the MR image acquisition is indeed also unknown. 
Based on [16], it has been already established that several combinations of $\rho, d_{w}, d_{g}$ lead to the same front speed and therefore the same tumor growth simulations. Therefore, it is sufficient in this inverse problem to consider a fixed value of the proliferation rate $\rho$ corresponding to the tumor grade and to estimate the remaining parameters. However, unlike $[15,16]$ this problem can be further simplified by realizing that the front speed cannot be estimated since $T_{\mathrm{Obs}}$ is unknown. If one multiplies the diffusivities by a scale factor $\alpha$ then one obtains the same isocontours for a propagation time divided by $\sqrt{\alpha}^{1}$. This means that the simulated tumor isocontours do not depend on that absolute value of $d_{g}$ and $d_{w}$ but on the diffusivity ratio:

$$
r=\frac{d_{w}}{d_{g}}
$$

In the remainder, we will show that this ratio is related to the spikiness of the tumor.

A simple sensitivity analysis has led to conclusion that solving the inverse tumor growth problem only depends on the following 2 parameters: the source location $S(\mathbf{x})$ and the spikiness index $r$. Here, the "spikiness index" represents a biology-driven estimated measure which quantifies the tortuousness of the boundary of the tumor as displayed on MR axial slices (see Figure 4-A), an index related to the frequency at which the tumor shape bends and twists. We consider that $\{S(\mathbf{x}), r\}$ appropriately characterize well a tumor extent if its visible boundary in MRI, $S_{\text {Seg }}$, is an isocontour of the simulated tumor growth initiated at $S(\mathbf{x})$ with diffusivity ratios equal to $r$. Therefore, we propose to estimate the patient specific parameters by minimizing the following criterion:

$$
C_{\rho}(S(x, y, z), r)=\frac{1}{N \bar{T}} \sqrt{\sum_{x \in S_{\mathrm{Seg}}}(T(x)-\bar{T})^{2}}
$$

with

$$
\bar{T}=\frac{1}{N} \sum_{x \in S_{\mathrm{Seg}}} T(x)
$$

\footnotetext{
${ }^{1}$ This is not strictly true if one uses the speed term $\mathrm{F}$ taking into account the transient speed as in Equation 4 or 5. However, the difference in simulations due to the absolute value of the diffusivities was found to be negligeable.
} 
where $N$ is the number of points belonging to the manually delineated tumor boundary $S_{\text {Seg }}$. In these equations, $\bar{T}$ and $C_{\rho}$ are respectively the mean time value and time standard deviation computed over the tumor boundary. Our motivation to use this criterion to get good estimates of our unknowns $(S(x, y, z), r)$ derives from the fact that a tumor boundary (propagating front) is simultaneously defined as an isotime and an isosurface. Thus, to quantify how good is the estimation of the parameters guiding the spatiotemporal evolution of the tumor shape, we need to quantify how closely the simulated isosurface matches the observed one (manually delineated boundary). From a time perspective, this also implies that when measuring the time $T$ at every point $x$ of the manually delineated tumor isotime boundary, its value $T(x)$ will be constant in the best case scenario where the simulated tumor front exactly matches the observed tumor boundary. Therefore, minimizing the time standard variation criterion $C_{\rho}$ over the delineated tumor boundary $S_{S e g}$ will guide the algorithm towards a better estimation of the two key parameters driving the invasive tumor front into fitting the successive MR observed boundaries. Note that $C_{\rho}$ is normalized by $\bar{T}$ because the criterion should be made independent of the tumor front speed and therefore the mean time $\bar{T}$.

In order to efficiently minimize the previously outlined criterion, we use the multidimensional unconstrained minimization algorithm without gradient introduced by Powell in [30]. This algorithm suits our case since our parameters are bounded in both biological and geometrical spaces. Moreover, the derivative of minimization criterion $C_{\rho}$ is not easy to compute. To better study the convergence of this algorithm and evaluate its outcome, 15 tests were performed using synthetic tumors. Further evaluation of this method was then studied using real data: two patients with LGG.

\subsection{Synthetic Data: synthetic tumor generation process}

In order to validate our parameter estimation, we produce synthetic tumor MR images, where the initial tumor location and diffusivity ratio are known. The procedure of generating synthetic tumors relies on choosing a seed point $S_{0}$ in either white or grey matter and the proliferation rate $\rho$ to one of the following values $0.008,0.012,0.024$ /day. The tumor front propagation is simulated with a white matter diffusion rate $d_{w}$ and grey matter diffusion rate $d_{g}$ whose ratio varies between 1 and 100 (considered to be a biologically valid range) using equation (5). The growth simulation is stopped at a specified time $T_{O b s}$ thus leading to a time distance map as seen in Fig 4. Different 
synthetic tumors were created at different anatomical locations with different sizes and asymptotic speeds of growth in both white and grey matter.

\section{Results}

\subsection{Convexity of the minimization function: a convergence study}

To check the convergence of the Powell minimization algorithm to the initially set parameters, we study the convexity of the minimization criterion $C$ (see Equation (6)) for the four scalar parameters $\left[S_{x}, S_{y}, S_{z}, r\right]$, writing separately the coordinates of the tumor source location. We proceed by alternatively fixing some parameters to their ground truth values and optimizing the remaining ones with the proposed minimization process. In Fig 5, we can clearly see the convexity of the minimization surface plotted after setting the diffusivity ratio $r$ and one of the source coordinates to their right values.

The convexity of the minimization criterion $C_{\rho}$ was successfully checked also when optimizing three parameters and setting the fourth one at its true value. The fact that the minimization criterion appears to be convex at the vicinity of the ground truth parameters is reassuring about the observability of the four parameters. However, the functional may still have local minima and practical optimization results will be discussed in the next sections.

\subsection{Synthetic data}

We evaluated our method on 15 synthetically generated tumors with a diffusivity ratio ranging from 1 to 100. The algorithm succeeded to identify the original tumor source with a mean error of $0.42 \mathrm{~mm}$ and a standard deviation of $0.36 \mathrm{~mm}$. Moreover, it always converges to the real diffusivity ratio $d_{w} / d_{g}$ with a mean error equal to 0.18 and a standard deviation of 0.06 .

Furthermore, we use this synthetic data to provide a better understanding of the diffusivity ratio $r$. The first row of Fig 6 shows how the shape of the tumor boundary can switch from "sphere-like" to "star-like" as the diffusivity ratio value jumps from 1 to 50. Although the displayed tumors in the third row are of different sizes, the correlation between tumors' shape spikiness and the value of the diffusivity ratio $r \in[1-100]$ is clear.

Besides, we also notice from the second row of Fig 6 similar tumor contours in terms of spikiness and irregularity when fixing the diffusivity ratio at the same value and varying the location of the initial tumor seed. This 
confirms that the diffusivity ratio value $r$ controls the spikiness of tumor shape.

\subsection{Clinical data}

\subsubsection{Evaluation criteria}

In clinical data, no ground truth values of the parameters are available and therefore additional criteria must be introduced in order to assess the quality of the parameter estimation.

After the optimization of criterion 6, we extract the simulated isocontour which is closest from the visible tumor boundary in MRI. Computing symmetric distances between the two surfaces provides a quantitative information about how well the tumor shape can be explained by the proposed tumor growth model. We detail below the proposed approach.

We extract the closest isocontour defined by time $\hat{T_{\text {Obs }}}$ by optimizing $C_{\text {isoTime }}(50 \%)(T)$ :

$$
\hat{T_{\text {Obs }}}=\min _{T} C_{\text {isoTime }}(50 \%)(T)
$$

The criterion $C_{\text {isoTime }}(50 \%)(T)$ is defined as the median symmetric distance between the visible tumour boundary $S_{\text {Seg }}$ and the isosurface $\hat{S}_{\text {isoTime }}$ at time $T$. More precisely, for each voxel of $\hat{S}_{\text {isoTime }}$ (resp. $S_{\text {Seg }}$ ) its closest distance from $S_{\text {Seg }}$ (resp. $\hat{S}_{\text {isoTime }}$ ) is computed through a distance map and added to a list. The median value of that list is then taken as $C_{\text {isoTime }}(50 \%)(T)$. The optimization of the functional is done with the Powell algorithm [30] already used for solving the general inverse problem and requiring few estimations of the functional. The range of time value for the optimization is constrained to be in the range $[\bar{T}-\delta ; \bar{T}-\delta]$ where $\delta$ is the standard deviation:

$$
\delta=\sqrt{\frac{\sum_{x \in S_{\mathrm{Seg}}}(T(x)-\bar{T})^{2}}{N}}
$$

We also compute other robust distance criteria $C_{\text {isoTime }}(y \%)(T)$ by taking the $y \%$ quantile of the symmetric distances.

Once the closest isochrone surface $\hat{S}_{\text {isoTime }}$ is estimated, we define the symmetric distance between $\left(S, \hat{S}_{\text {isoTime }}\right)$ as the first evaluation criterion: 
$C_{\text {symDist }}\left(S_{\text {Seg }}, \hat{S}_{\text {isoTime }}\right)=\frac{\sum_{\mathbf{x} \in S_{\text {Seg }}} \min d\left(\mathbf{x}, \hat{S}_{\text {isoTime }}\right)+\sum_{\mathbf{x} \in \hat{S}_{\text {isoTime }}} \min d\left(\mathbf{x}, S_{\text {Seg }}\right)}{\left|S_{\text {Seg }}\right|+\left|\hat{S}_{\text {isoTime }}\right|}$

where $d$ denotes the closest Euclidean distance between a point and a surface.

We can also measure the similarity between the visible tumor volume $V_{\text {seg }}$ and the simulated tumor volume $V_{\text {isoTime }}$ :

$$
C_{\text {overlap }}\left(V_{\text {seg }}, V_{\text {isoTime }}\right)=\frac{V_{\text {seg }} \cap V_{\text {isoTime }}}{0.5\left(V_{\text {seg }}+V_{\text {isoTime }}\right)}
$$

\subsubsection{Study of Patient A}

For patient studies, we take advantage of the fact that several FLAIR MR images are available for different time points. We first check the stability of source locations for the $M$ time points per patient and correlate the spikiness index with the observed tumor evolution. To estimate the two target parameters $[S(\mathbf{x}), r]$, the proliferation rate was fixed to $0.012 /$ day. Table 1 represents the outcome of the estimation method for the four time points $\left(t_{1}\right.$, $\left.t_{2}, t_{3}, t_{4}\right)$ of Patient $\mathrm{A}$.

\begin{tabular}{ccccc}
\hline & $t_{1}$ & $t_{2}$ & $t_{3}$ & $t_{4}$ \\
\hline$S(\mathbf{x})$ & $(120,111,31)$ & $(120,111,31)$ & $(122,115,30)$ & $(121,116,30)$ \\
\hline$d(G, S)$ in $\mathrm{mm}$ & 2.00 & 1.73 & 1.42 & 5.38 \\
\hline$r$ & 41.98 & 40.47 & 40.58 & 41.38 \\
\hline
\end{tabular}

Table 1: Outcome of the minimization algorithm of Patient A which was separately computed at four time points. The distance $d$ denotes the Euclidean distance between the tumor barycenter $G$ and its estimated source $S$.

We can clearly observe in Table 1 the stability of the tumor source location and the fact that for the first 3 time points this location is very close to the tumor barycenter since the Euclidean distance between them $d(G, S)$ ranges from $1.42 \mathrm{~mm}$ to $2 \mathrm{~mm}$. Nonetheless, for the fourth time point, the estimated tumor source distance from its barycenter is $5.38 \mathrm{~mm}$. Moreover, the diffusion rate $r$ is mainly constant. In fact, patient A showed a very slow and quasistatic evolution of the tumor boundary between the second and the third acquisition time points. Our simulation model depicts this static evolution 


\begin{tabular}{lcc}
\hline & $C_{\text {symDist }}$ in $\mathrm{mm}$ & $C_{\text {overlap }}$ in $\%$ \\
\hline$\left(\right.$ Patient $\left.A, t_{2}\right)$ & 1.97 & 85.63 \\
\hline (Patient $\left.A, t_{3}\right)$ & 1.79 & 87.76 \\
\hline$\left(\right.$ Patient $\left.A, t_{4}\right)$ & 1.74 & 88.04 \\
\hline (Patient,$\left.t_{2}\right)$ & 1.53 & 82.20 \\
\hline (PatientB, $\left.t_{3}\right)$ & 1.48 & 84.79 \\
\hline (Patient,$\left.t_{4}\right)$ & 1.13 & 88.02 \\
\hline
\end{tabular}

Table 2: Prediction evaluation based on the error measures $C_{\text {symDist }}$ and $C_{\text {overlap }}$ computed for Patient A and B to predict the evolution of the tumor at specific time points $t_{i}$ using the minimization outcome of the previous time $t_{i-1}$.

behavior as the corresponding estimated spikiness index $r$ shown in Table 1 remains quasi-static $\left(r=40.47\right.$ at $t_{2}$ and 40.58 at $\left.t_{3}\right)$, which is in line with the identical appearance of the estimated tumor red boundaries shown in the first and second rows of Figure 7. However, it is worth noting that the algorithm failed to catch the tiny bump that appeared in the tumor boundary at $t_{3}$ (see first column, row 1 and 2 in Figure 7). This can be explained by the fact that the algorithm is based on a mean error minimization process, which therefore accounts for a mean morphological change in tumor shape over all the axial slices included in the tumor volume.

Second, we study the possibility to predict further spatial tumor boundaries evolution based on parameter evaluation. More precisely, after estimating patient specific parameters at time $t_{i}$, we run a forward simulation using those estimated parameters trying to predict the tumor invasion process as time evolves. We then proceed by estimating the closest predicted isocontour from the observed tumor boundary at time $t_{i+k}$ as described in section3.3.1. Fig 8 and Table 2 display the outcomes of the evaluation criteria for every time point.

We notice a limited discrepancy between the observed contour at time point $t_{i}$ and the predicted one at $t_{i+1}$ since the symmetric distance between these contours $C_{\text {symDist }}$ ranges from $1.74 \mathrm{~mm}$ to $1.97 \mathrm{~mm}$. Moreover the median distance error $C_{\text {isoTime }}(50 \%)$ ranges from $1.44 \mathrm{~mm}$ to $1.68 \mathrm{~mm}$ and the volume overlap $C_{\text {overlap }}$ between the real tumor volume and the extracted one ranges from $85.63 \%$ to $88.04 \%$. It is remarkable that the estimated parameters at a specific time point $t_{i}$ captures the tumor spatial evolution at the successive time point $t_{i+1}$. As highlighted in Fig 8, the estimated- 


\begin{tabular}{ccccc}
\hline & $t_{1}$ & $t_{2}$ & $t_{3}$ & $t_{4}$ \\
\hline$S(\mathbf{x})$ & $(34,145,117)$ & $(35,141,117)$ & $(34,141,118)$ & $(34,140,119)$ \\
\hline$d(G, S)$ in $\mathrm{mm}$ & 5.09 & 5.47 & 5.09 & 4.24 \\
\hline$r$ & 6.45 & 37.15 & 30.12 & 47.70 \\
\hline
\end{tabular}

Table 3: Outcome of the minimization algorithm of Patient B separately computed at four time points.

parameters based prediction shows promising results.

\subsubsection{Study of Patient B}

The parameter estimation outcome for Patient B at its four time points is summarized in Table 3. The obtained results confirm the stability of the tumor source location. At every time point, the distance between the tumor barycenter and the located source exceeds $4 \mathrm{~mm}$, meaning that the tumor source may not be close to its barycenter. Unlike Patient A, the spikiness index value $r$ increases as time evolves as it can also assessed visually in Fig 9.

Despite the poor signal quality of the DT-MRI, the evaluation criteria confirm that the estimated parameters were adequate to spatially predict the tumor front propagation. In fact, the symmetric distance $C_{\text {symDist }}$ between the real front observed at $t_{i}$ and the extracted one at $t_{i+1}$ ranges from $1.13 \mathrm{~mm}$ to $1.53 \mathrm{~mm}$. The volume overlap $C_{\text {overlap }}$ ranges from $82.20 \%$ to $88.02 \%$ (Table 2$)$. The median distance error $C_{\text {isoTime }}(50 \%)$ ranges from 0.92 $\mathrm{mm}$ to $1.16 \mathrm{~mm}$ (Fig 8). Fig 10 shows that the algorithm succeeded to spatially capture the geometric behavior of the evolving tumor. However, the maximum distance error $C_{\text {isoTime }}(100 \%)$ reaches high values ranging from $7.10 \mathrm{~mm}$ to $11.97 \mathrm{~mm}$. These outliers are related to the interpolated holes in the DT-MRI where the simulated propagating front fails to capture the real tumor invasion process.

\subsubsection{Study of Patients $C$ and $D$}

In both patient $\mathrm{C}$ and $\mathrm{D}$, the estimated source remained stable from the first time point to the third one as the distance between the successively estimated sources $d\left(S_{t_{1}}, S_{t_{2}}\right)$ and $d\left(S_{t_{1}}, S_{t_{3}}\right)$ did not reach a value over $1.42 \mathrm{~mm}$. The estimated diffusivity ratio for Patient $\mathrm{C}$ remained constant as time evolves $(r=7.92)$, a spikiness value in concordance with the round 
shape of the tumor boundary and its very slow evolution between $t_{1}$ and $t_{3}$ as shown in Fig 11. The spatial disparity between the estimated source and the barycenter did not exceed $1.8 \mathrm{~mm}$. For Patient D, the estimated diffusivity ratio varied from $r_{t_{1}}=20$ to $r_{t_{2}}=16.2$ then increased to $r_{t_{3}}=23.8$. The successively estimated high diffusivity ratios are in line with the tumor boundary spikiness shown in Fig 2-b. The computed distance between the estimated source and the tumor barycenter varied from $1.42 \mathrm{~mm}$ at $t_{1}$ to $3 \mathrm{~mm}$ at both $t_{2}$ and $t_{3}$.

\section{Discussion}

The patient-specific parameter estimation method presented in this paper uses two main ingredients: the traveling front approximation presented by Konukoglu et al. [16] transformed into the AEE and the Powell minimization algorithm. We have shown using a limited number of LGG cases that our approach could provide clinicians with two fundamental tumor dynamics characteristics: the spatial position of tumor seed point and its diffusivity ratio. Our optimization approach has been successfully evaluated on synthetic data where ground truth is available.

At this point, we have noticed the quasi-stability of the located source in both clinical cases, which is reassuring. Another key finding is related to the distance between the located source point and tumor barycenter. For Patient B, this distance ranges from $4.24 \mathrm{~mm}$ to $5.47 \mathrm{~mm}$ and for the 15 synthetically generated tumors, computed distance ranges from $1.42 \mathrm{~mm}$ to over $13 \mathrm{~mm}$. The disparity between the computed source and its barycenter maybe explained by the spikiness index. Indeed, it is intuitive that "spherelike" tumors have a source which might be very close to its barycenter but "star-like" tumors or spiky tumors are prone to greater disparities. In the latter case, the barycenter may even end-up outside the tumor shape. This outcome contradicts the assumption that the tumor source is its barycenter as assumed in some studies as in [23] (tumor classification process based on the correlation between its located barycenter and molecular profile ), in [24] (MGMT gene methylation status prediction study in glioblastomas) and in $[25,26,27]$ (tumor growth simulation using cancerous cell concentration gradient computed from the center of tumor mass). Therefore, tumor barycenter-derived conclusions may not seem fully reliable and could cloud the true biological phenomena influencing tumor growth patterns. Furthermore, as noticed in Table 1, the source-to-barycenter distance ranging from 1 
to $5 \mathrm{~mm}$ in a tumor of $10 \mathrm{~mm}$-size demonstrates that choosing the barycenter as a source estimator leads to a large bias.

In Patient A, there is a jump of the source-barycenter distance value from $1.42 \mathrm{~mm}$ to $5.38 \mathrm{~mm}$. This may be explained by a recent study from Bohman et al. [31] aiming at a better understanding of glioma ontogeny (development from earlier stage) within the complexity of brain anatomy. In fact, Bohman et al. concluded that as the tumor grows, its boundary approaches the ventricles and the closer its "seed position" is to the ventricles, the larger the tumor would be. This conclusion agrees with our observation in Fig 7 where the tumor boundary becomes more distorted as it seems to be more "pulled" towards the left lateral ventricle. Note that while Bohman et al. use a basic geometric method to estimate the tumor barycenter, it mentions a discrepancy between barycenter and tumor source ranging between $1.25 \mathrm{~mm}$ and 15 $\mathrm{mm}$. This is in agreement with our synthetic study and we hope that this work could provide a better understanding of tumor ontogeny.

According to our study, the tumor shape irregularities seems to be well correlated with the diffusivity ratio $r$. Setting the spikiness index leads to similar irregular tumor contours up to the geometry of their neighboring brain structures (presence of the ventricles for instance). As a matter of fact, the quasi-stability of this ratio for Patient $\mathrm{A}$ is justified by the quasistability of its shape over time. Its high values ranging from 40.5 to 42 agrees with its "V-like" irregularity (Fig 7). On the other hand, for Patient B, this ratio raises from 6.45 to 47.70 as its shape evolves from a "sphere-like" to a "star-like" (Fig 9). Interestingly, in a recent paper [32], a different ratio $\left(d_{w} / \rho\right)$ was used to quantify the diffusivity of the tumor boundary where a low white matter diffusivity rate over proliferation ratio indicates a not very infiltrative glioma (bulky), whereas high ratio defines diffuse tumor. The main objective was to determine whether the tumor recurrence is a bulky or diffuse-type recurring tumor. We believe that a comparison between both of these ratios $\left(d_{w} / d g\right.$ and $\left.d_{w} / \rho\right)$ in future research works will be a valuable step in determining the patient-specific factors guiding tumor growth.

For patients $\mathrm{C}$ and D, which had DT-MRI anomalies as shown in (Fig 1 and 2), our method proved its robustness as the estimated tumor source remained stable as time evolved. The symmetrization process induced a tremendous loss in white matter anisotropy as most of the fibers along which the tumor originally grew disappeared as demonstrated in (Fig 1) through FA histograms comparison. Nevertheless, the algorithm converged to a low diffusivity ratio value $(r=7.92)$. That was visually proven to be in con- 
cordance with the tumor "roundness" (Fig 11) and also remained constant throughout time, a fact in correlation with the very slow and slightly unchanged tumor boundary from $t_{1}$ to $t_{3}$. The Euclidean distance between the estimated tumor source and its barycenter did not go over $2 \mathrm{~mm}$, which was quite expected when noticing the roundness of the tumor shape.

Patient D did not undergo the symmetrization process because of the large holes in the symmetric region to tumor segmented tissue (Fig 2-a) and the original diffusion fibers distorted by tumor growth were used. Despite this, the estimated source remained stable with $1 \mathrm{~mm}$ spatial variation along z-axis. Its spatial disparity to the barycenter reaching a value of $3 \mathrm{~mm}$ highlights that the increase of the tortuosity of the tumor quantified by the estimated diffusivity ratio implies an increase in the distance between the tumor barycenter and its original source (as in Patient B where it reached $5.47 \mathrm{~mm}$ ). Since a good estimation of the spikiness index is tied to the good quality of the acquired DT-MRI, the unexpected drop in $r$ value from $r_{t_{1}}=20$ to $r_{t_{2}}=16.2$ can be explained by the major tumor-infiltration derived anomalies of the reconstructed diffusion tensors and also the interpolation of the small holes therein. Both cases with various DTI anomalies demonstrated a good performance when estimating a stable tumor source and also capturing the spikiness of its shape through the estimated diffusivity ratio. However, the prediction step was overlooked since it strongly requires a very good quality of the symmetric DTI to the affected invaded tissue.

Relying on key biological tissue characteristic (diffusivity in white and grey matters), our estimation of the tumor source location and diffusivity rate (or spikiness index) also showed a promising prediction of the further spatial propagation of the glioma boundary as time evolves. The good prediction in both LGG cases presented in Table 2 and Fig 7 provides additional confidence in the estimation of tumor source and diffusivity ratio. Note however that this spatial prediction was not based on a simulation from the tumor origin but from the tumor boundary at a previous time point using the estimated spikiness index. This is to avoid error accumulation leading to a weak prediction.

DT-MRI serves as the basis for several reaction-diffusion based models to extrapolate glioma invasion margins for radiation [33] and after resection [32] using at least two acquired time points. Those two studies have been successively evaluated on one healthy subject and one LGG patient, and they confirm that the importance of using high resolution and high quality DTMRI which is obviously a strong constraint. Including DT-MRI acquisitions 
in a standardized brain tumor imaging protocol would increase the number of recruited patients and could allow a thorough validation of these models.

\section{Conclusion}

To go beyond the classical tumor radial expansion model which does not take into account the tumor boundary irregularities ([31], [34], [35]), we have introduced a new method to estimate tumor growth parameter based on a traveling front propagation framework. The described method provides two main patient-specific spatial characteristics of a tumor using one clinical image at a single time point: its source location and an index quantifying the irregularity of its shape. The proposed macro-scale approach was evaluated using synthetic data and four patients with LGG. As a consequence of the estimation of both parameters, a promising prediction of tumor spatial behavior was pointed out.

Some aspects of this work may be subject to improvements. For instance, a sensitivity analysis of the proposed method to the choice of tumor diffusion tensor in white matter is needed. Our proposed formulation leads to efficient computations, but a quantitative evaluation from experimental datasets should help refining the approach. Also, the white matter and brain mask segmentations could be improved by using state-of-the-art image classification tools on high resolution T1 MRI for instance.

Future steps include the application of the proposed method on highgrade gliomas and on a large database of glioma patients advancing a better validation of the predictive power previously highlighted. This could also lead to a statistical study of the correlation between the tumor source location, its grade and its diffusivity ratio. Thus, we could check whether lower spikiness index values are obtained for HGG since they appear to have less spiky and irregular shapes than LGG. Including the mass effect when addressing HGG growth would be a necessary step to include in the presented methodology. Hopefully, the coupling of mathematical models with clinical images should lead to a better understanding of tumor growth and to useful computational tools for diagnosis and therapy planning.

\section{Acknowledgments}

We thank Ender Konukoglu for his assistance in understanding his research work previously referenced. This work was partially funded by the ERC advanced Grant MedYMA. 
[1] M. Tovi. MR imaging in cerebral gliomas analysis of tumour tissue components. Acta radiologica. Supplementum, 384:1, 1993.

[2] E. Bearer, J. Lowengrub, H. Frieboes, Y. Chuang, F. Jin, S. Wise, M. Ferrari, D. Agus, and V. Cristini. Multiparameter computational modeling of tumor invasion. Cancer research, 69(10):4493, 2009.

[3] H. Frieboes, J. Lowengrub, S. Wise, X. Zheng, P. Macklin, E. Bearer, and V. Cristini. Computer simulation of glioma growth and morphology. Neuroimage, 37:S59-S70, 2007.

[4] S. Sanga, H. Frieboes, X. Zheng, R. Gatenby, E. Bearer, and V. Cristini. Predictive oncology: a review of multidisciplinary, multiscale in silico modeling linking phenotype, morphology and growth. Neuroimage, 37:S120-S134, 2007.

[5] C. Wang, J. Rockhill, M. Mrugala, D. Peacock, A. Lai, K. Jusenius, J. Wardlaw, T. Cloughesy, and A. SpenceR. Rockne. Prognostic significance of growth kinetics in newly diagnosed glioblastomas revealed by combining serial imaging with a novel biomathematical model. Cancer research, 69(23):9133, 2009.

[6] J.D. Murray. Mathematical biology, volume 2. Springer, 2002.

[7] K. Swanson, E. Alvord, and J. Murray. A quantitative model for differential motility of gliomas in grey and white matter. Cell Proliferation, 33(5):317-330, 2000.

[8] A. Giese, L. Kluwe, B. Laube, H. Meissner, M. Berens, M. Westphal, E. Laws, K. Edvardsen, M. Rosenblum, and J. Rutka. Migration of human glioma cells on myelin. commentaries. Neurosurgery, 38(4):755764, 1996.

[9] S. Jbabdi, E. Mandonnet, H. Duffau, L. Capelle, K. Swanson, M. Pélégrini-Issac, R. Guillevin, and H. Benali. Simulation of anisotropic growth of low-grade gliomas using diffusion tensor imaging. Magnetic Resonance in Medicine, 54(3):616-624, 2005.

[10] Olivier Clatz, Maxime Sermesant, Pierre-Yves Bondiau, Hervé Delingette, Simon K. Warfield, Grégoire Malandain, and Nicholas Ayache. Realistic simulation of the $3 \mathrm{~d}$ growth of brain tumors in $\mathrm{mr}$ im- 
ages coupling diffusion with mass effect. IEEE Transactions on Medical Imaging, 24(10):1334-1346, October 2005.

[11] E. Angelini, O. Clatz, E. Mandonnet, E. Konukoglu, L. Capelle, and H. Duffau. Glioma dynamics and computational models: a review of segmentation, registration, and in silico growth algorithms and their clinical applications. Current Medical Imaging Reviews, 3(4):262-276, 2007.

[12] K. Swanson, C. Bridge, J. Murray, and E. Alvord. Virtual and real brain tumors: using mathematical modeling to quantify glioma growth and invasion. Journal of the neurological sciences, 216(1):1-10, 2003.

[13] T. Colin, A. Iollo, and D. Lombardi. System identification in tumor growth modeling using semi-empirical eigenfunctions. Mathematical Models and Methods in Applied Sciences, 22(06):1250003, 2012.

[14] E. Konukoglu, M. Sermesant, O. Clatz, J.-M. Peyrat, H. Delingette, and N. Ayache. A recursive anisotropic fast marching approach to reaction diffusion equation: Application to tumor growth modeling. In Proceedings of the 20th International Conference on Information Processing in Medical Imaging (IPMI'O'7), volume 4584 of LNCS, pages 686-699, 2-6 July 2007.

[15] E. Konukoglu, O. Clatz, P. Bondiau, M. Sermesant, H. Delingette, and N. Ayache. Towards an identification of tumor growth parameters from time series of images. Medical Image Computing and Computer-Assisted Intervention, MICCAI 200\%, pages 549-556, 2007.

[16] E. Konukoglu, O. Clatz, B. Menze, B. Stieltjes, M. Weber, E. Mandonnet, H. Delingette, and N. Ayache. Image guided personalization of reaction-diffusion type tumor growth models using modified anisotropic eikonal equations. Medical Imaging, IEEE Transactions on, 29(1):77-95, 2009 .

[17] L.M. Deangelis. Brain tumors. New England Journal of Medicine, 344(2):114-123, 2001.

[18] E.C.A. Kaal and C.J. Vecht. The management of brain edema in brain tumors. Current opinion in oncology, 16(6):593, 2004. 
[19] I. Whittle. The dilemma of low grade glioma. Journal of Neurology, Neurosurgery $\&$ Psychiatry, 75(suppl 2):ii31-ii36, 2004.

[20] A. Gooya, G. Biros, and C. Davatzikos. Deformable registration of glioma images using em algorithm and diffusion reaction modeling. IEEE Transactions on Medical Imaging, 30(2):375-390, 2011.

[21] A. Gooya, K. Pohl, M. Bilello, G. Biros, and C. Davatzikos. Joint segmentation and deformable registration of brain scans guided by a tumor growth model. In Medical Image Computing and Computer-Assisted Intervention, MICCAI 2011, volume 6892 of LNCS, pages 532-540. Springer, 2011.

[22] A. Gooya, K. Pohl, M. Bilello, L. Cirillo, G. Biros, E. Melhem, and C. Davatzikos. GLISTR: Glioma image segmentation and registration. IEEE Transactions on Medical Imaging, 31(10):1941-1954, 2012.

[23] F. Laigle-Donadey, N. Martin-Duverneuil, J. Lejeune, E. Criniere, L. Capelle, H. Duffau, P. Cornu, P. Broet, M. Kujas, and K. Mokhtari. Correlations between molecular profile and radiologic pattern in oligodendroglial tumors. Neurology, 63(12):2360, 2004.

[24] S. Drabycz, G. Roldán, P. De Robles, D. Adler, J. Mcintyre, A. Magliocco, and J. Cairncross. An analysis of image texture, tumor location, and mgmt promoter methylation in glioblastoma using magnetic resonance imaging. Neuroimage, 49(2):1398-1405, 2010.

[25] A.R.A. Anderson. A hybrid mathematical model of solid tumour invasion: the importance of cell adhesion. Mathematical Medicine and Biology, 22(2):163-186, 2005.

[26] A. Sottoriva, J.J.C. Verhoeff, T. Borovski, S.K. Mcweeney, L. Naumov, J.P. Medema, P. Sloot, and L. Vermeulen. Cancer stem cell tumor model reveals invasive morphology and increased phenotypical heterogeneity. Cancer research, 70(1):46, 2010.

[27] A. Kansal, S. Torquato, E. Chiocca, and T. Deisboeck. Emergence of a subpopulation in a computational model of tumor growth. Journal of Theoretical Biology, 207(3):431-441, 2000. 
[28] G.S. Young. Advanced mri of adult brain tumors. Neurologic clinics, 25(4):947-973, 2007.

[29] P. Tracqui, G. Cruywagen, D. Woodward, G. Bartoo, and J. MurrayE.C. Alvord Jr. A mathematical model of glioma growth: the effect of chemotherapy on spatio-temporal growth. Cell Proliferation, 28(1):1731, 1995.

[30] M. Powell. The bobyqa algorithm for bound constrained optimization without derivatives. Cambridge NA Report NA2009/06, University of Cambridge, Cambridge, 2009.

[31] L.E. Bohman, K.R. Swanson, J.L. Moore, R. Rockne, C. Mandigo, T. Hankinson, M. Assanah, P. Canoll, and J.N. Bruce. Magnetic resonance imaging characteristics of glioblastoma multiforme: implications for understanding glioma ontogeny. Neurosurgery, 67(5):1319, 2010.

[32] Erin Stretton, Emmanuel Mandonnet, Ezequiel Geremia, Bjoern H. Menze, Herve Delingette, and Nicholas Ayache. Predicting the location of glioma recurrence after a resection surgery. In Proceedings of 2nd International MICCAI Workshop on Spatiotemporal Image Analysis for Longitudinal and Time-Series Image Data (STIA'12), LNCS, Nice, October 2012. Springer.

[33] E. Konukoglu, O. Clatz, P.Y. Bondiau, H. Delingette, and N. Ayache. Extrapolating glioma invasion margin in brain magnetic resonance images: Suggesting new irradiation margins. Medical image analysis, 14(2):111-125, 2010.

[34] E. Mandonnet, J.Y. Delattre, M.L. Tanguy, K.R. Swanson, A.F. Carpentier, H. Duffau, P. Cornu, R. Van Effenterre, E.C. Alvord, and JrL. Capelle. Continuous growth of mean tumor diameter in a subset of grade ii gliomas. Annals of neurology, 53(4):524-528, 2003.

[35] H. Harpold, E.C. Alvord, and Jr.K. Swanson. The evolution of mathematical modeling of glioma proliferation and invasion. Journal of Neuropathology \& Experimental Neurology, 66(1):1, 2007. 


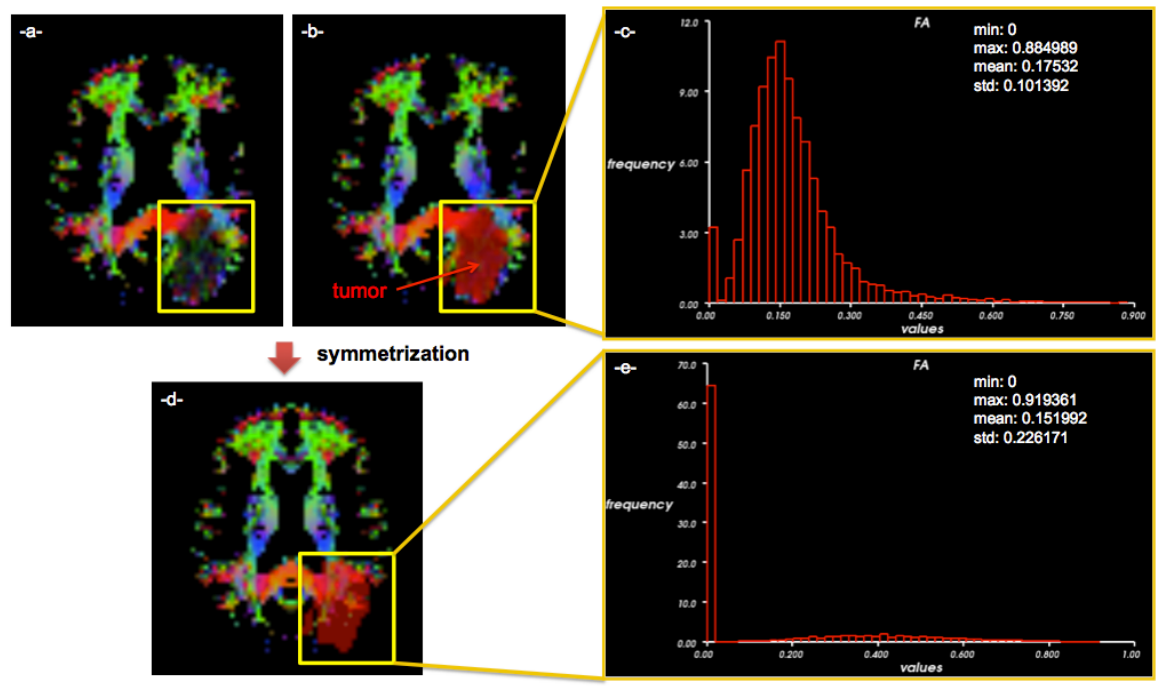

Figure 1: Data encountered problems (Patient C): fractional anisotropy (FA) asymmetry. (a): Non-symmetrized FA color map shows the dramatic diffusion signal distortion as the region where the tumor grew seems darker than the unaffected brain tissue. (b): Nonsymmetrized FA color map overlayed on glioma segmentation. (d) Symmetrized FA color map overlayed on glioma segmentation. A preliminary visual comparison between (b) and (d) clearly shows that when symmetrizing the diffusion tensor image and therefore the driven FA map, there is a tremendous loss in white matter anisotropy as most of the fibers along which the tumor originally grew disappear. The comparison of FA histograms only computed in the segmented tumor region (c) and (d) successively corresponding to (b) and (d) tumor figures demonstrates the anisotropy bias introduced by symmetrization process in this case. 

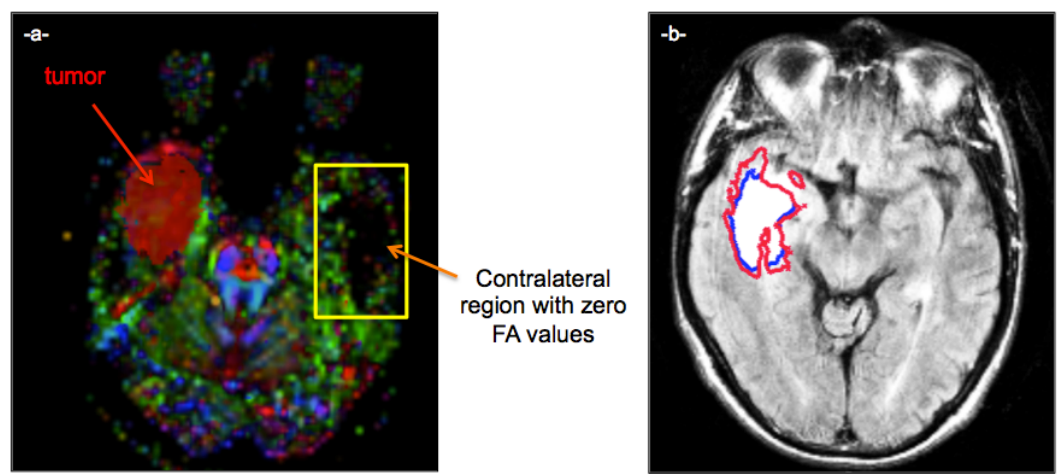

Figure 2: Data encountered problems (Patient D): large black holes in the DTI. (a) Tumor segmentation in red is overlayed on the fractional anisotropy map, a measure derived from Diffusion Tensor Imaging. The significantly missing diffusion signal in the contralateral region to tumor segmented tissue prohibited our proceeding to the symmetrization process and therefore we used for our estimation the affected tissue tensors by tumor infiltration. (b) The tumor boundary is drawn on the FLAIR image at the first and second time points in light and dark blue (in this slice they are identical) and at the third time point in red. 

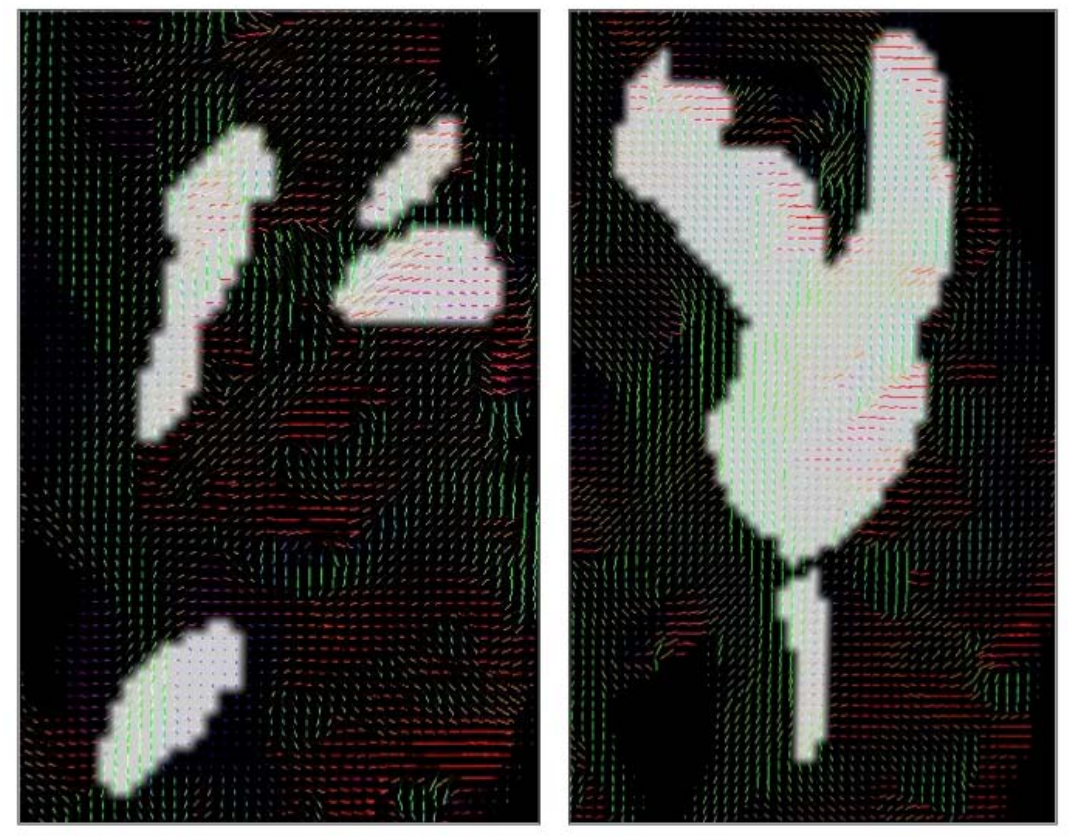

Figure 3: Two axial slices of the first included patient (Patient A) where the manually segmented glioma (in white) is visualized on the diffusion tensor MR image. The symmetrization process in this case led to reliably recovered infiltration fiber tracts where the simulation of tumor growth starting from a seed point is legitimate. 


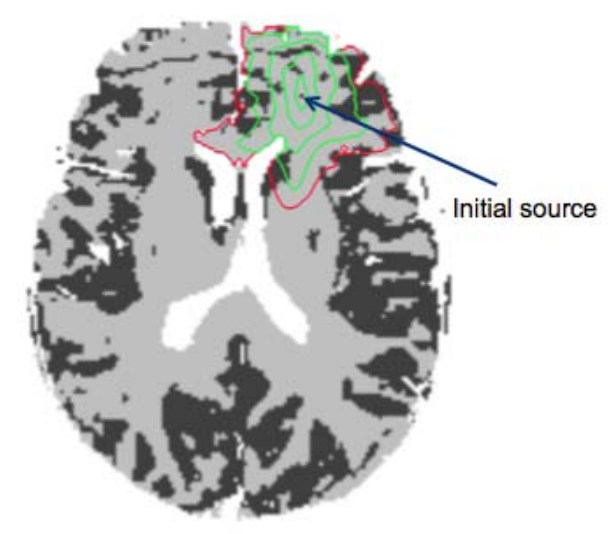

(A)

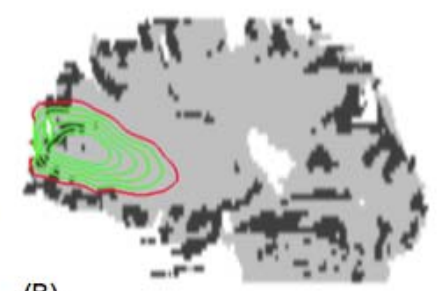

(B)

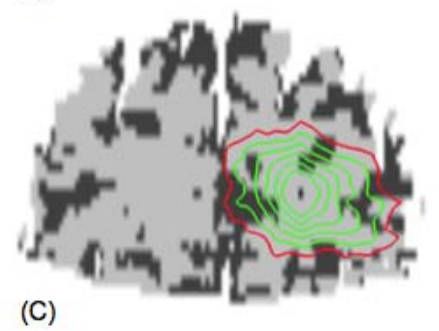

Figure 4: Simulation from a source point of the iso-time contours representing the tumor invasion process with a diffusivity ratio $d_{w} / d_{g}$ equal to 25 . The synthetic tumor displayed in red is created by thresholding the time distance map at $T=T_{\text {Obs }}$. (A) represents the axial slice, (B) coronal slice and (C) sagittal slice. 


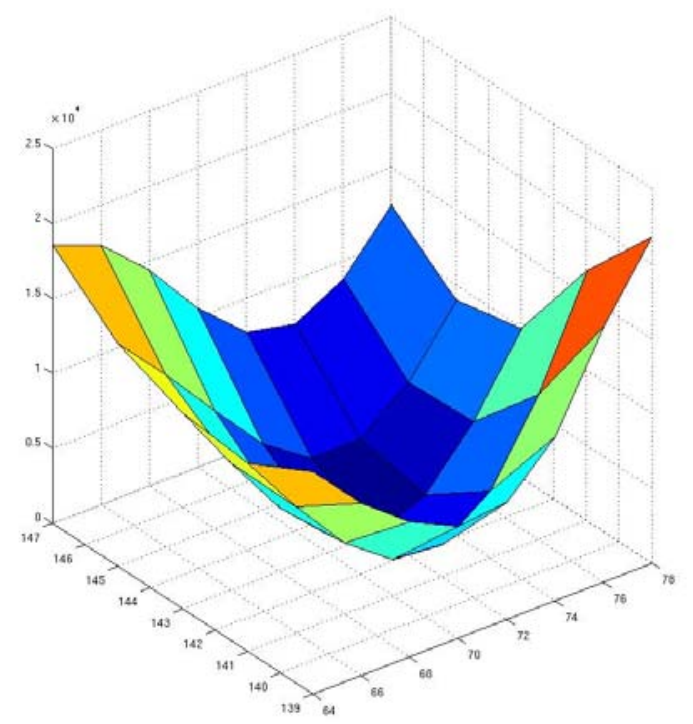

Figure 5: The minimization surface after fixing $r$ and $S_{z}$. The $x$ axis of this 3D plot represents $x$ coordinate of tumor source and the $y$ axis represents its $y$ coordinate. 

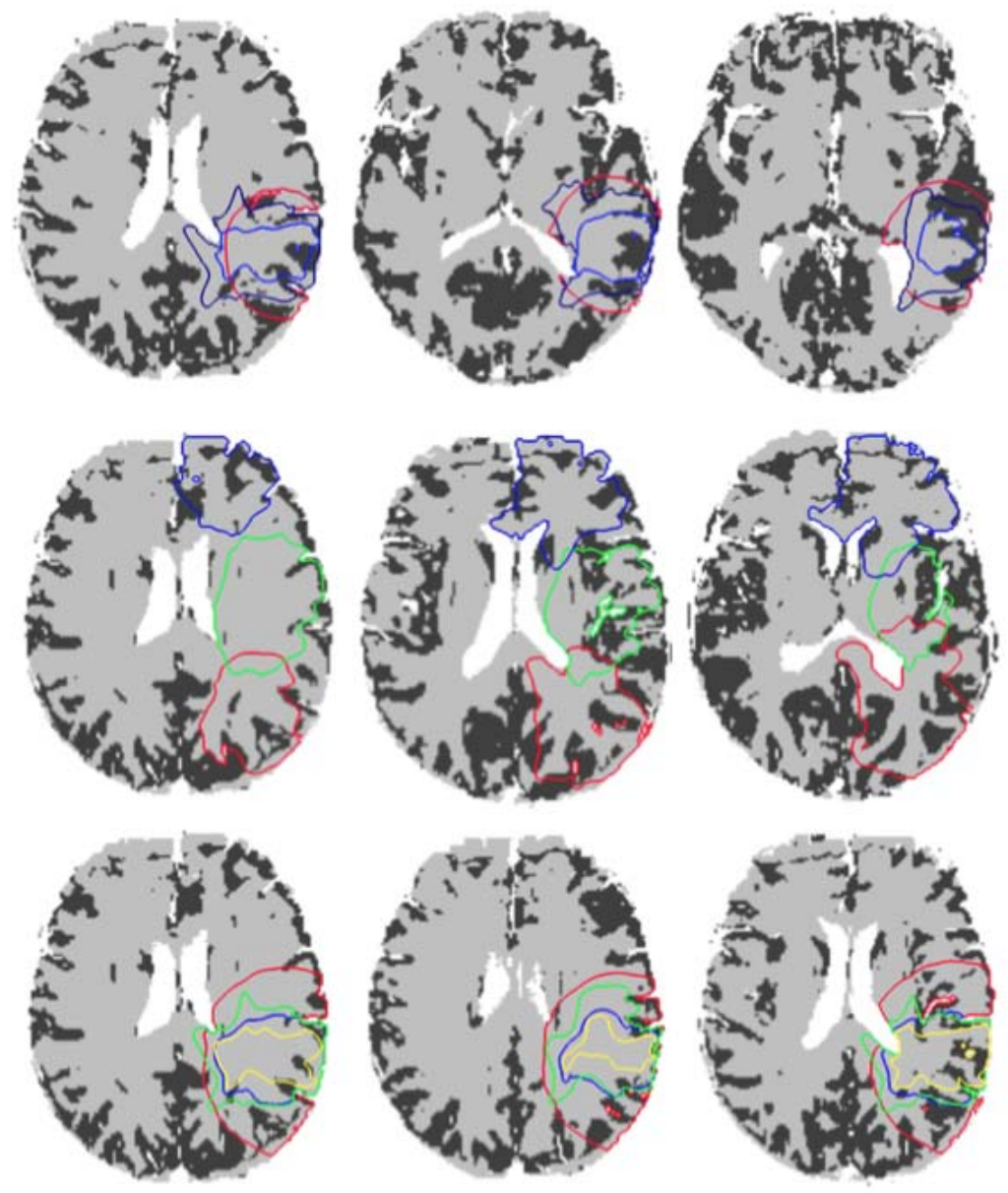

Figure 6: Synthetic tumors generated at different locations with different diffusivity ratios and observation times. First row: At a fixed source location, two iso-time contours with the same observation time $\left(T_{O b s}=900\right.$ days $)$ are respectively colored in red for a diffusivity ratio $(r=1)$ and in light blue $(r=50)$. The darker blue contour represents the tumor further spatial invasion with $(r=50)$ and $\left(T_{f}=1200\right.$ days $)$. Second row: 3 synthetic tumors are generated using the same observation time value $T_{O b s}$ and the same diffusivity ratio $(r=25)$ for 3 different source locations. Third row: At the same fixed location, five iso-time contours with the same observation time are colored in red $(r=1)$, in blue $(r=10)$, in green $(r=50)$ and in yellow $(r=100)$. 

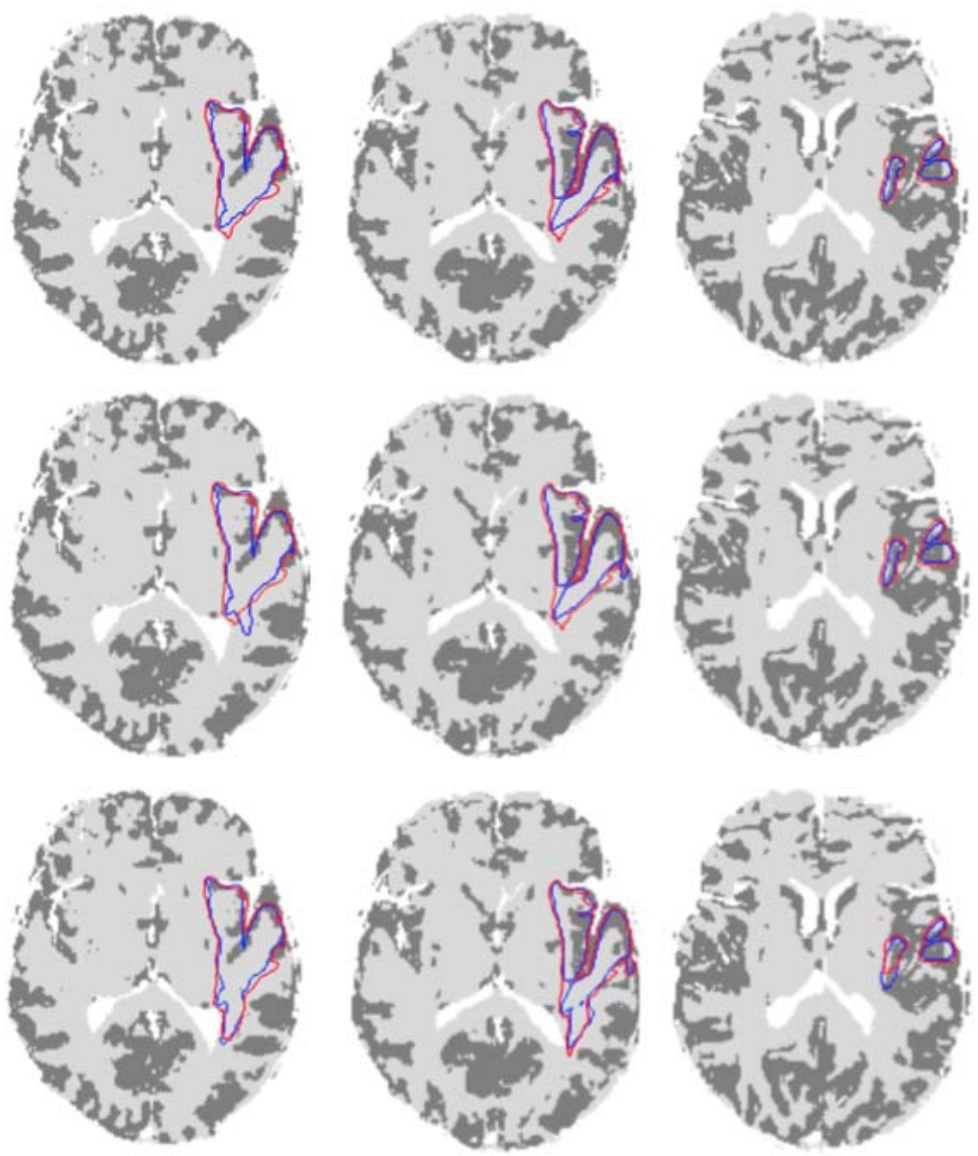

Figure 7: Different axial slices of Patient A showing the discrepancy between the real boundary and the estimated one at 3 different successive time points. First row: using the algorithm outcome at $t_{1}$, we spatially determine the tumor evolution at $t_{2}$. The blue contour represents the manually delineated tumor at the second time point and the red one is the extracted iso-time surface $\hat{S}_{\text {isoTime }}$. Second row: using the algorithm outcome at $t_{2}$, we predict the tumor evolution at $t_{3}$. Tumor boundary at $t_{3}$ is colored in blue and the estimated one in red. Third row: using the algorithm outcome at $t_{3}$, we predict the tumor evolution at $t_{4}$. Same colors are used to show the similarity between the estimated and the ground-truth tumor contours. 


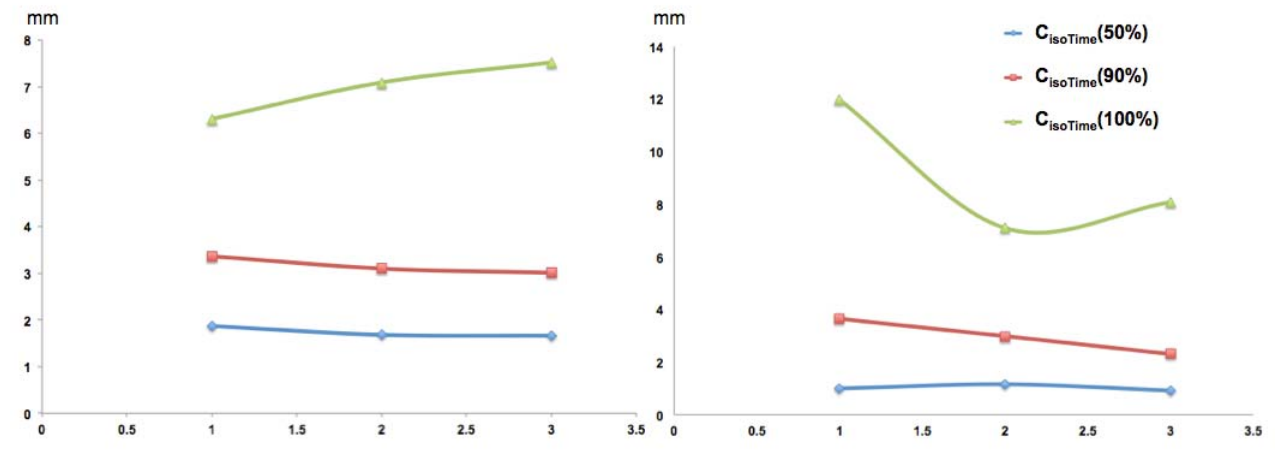

Figure 8: Evaluation curves plotted using the distance error measure $C_{\text {isoTime }}(y \%)$ for $y \in(50,90,100)$. For each patient (A or B), we try the predict the evolution at a successive time point $\left(A / B, t_{i}\right)$ based on the estimated parameters at the previous one.
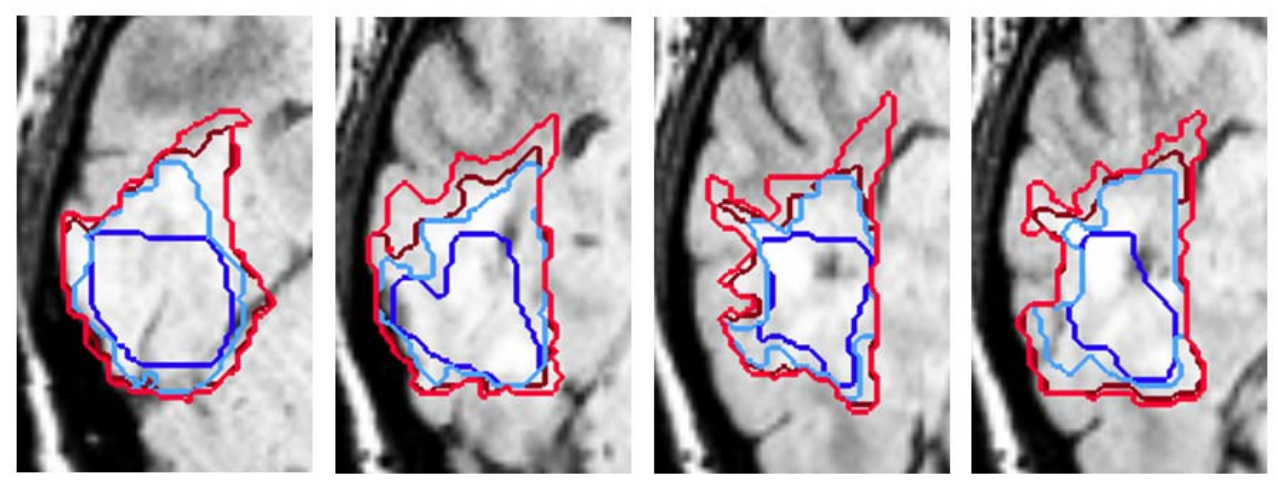

Figure 9: Four different axial slices of Patient B showing the remarkable tumor boundary spikiness evolution from the initial time point $t_{1}$ to the final one $t_{4}$. Tumor contours at successive time points are respectively colored in: dark blue for $t_{1}$, light blue for $t_{2}$, dark red for $t_{3}$ and light red for $t_{4}$. 

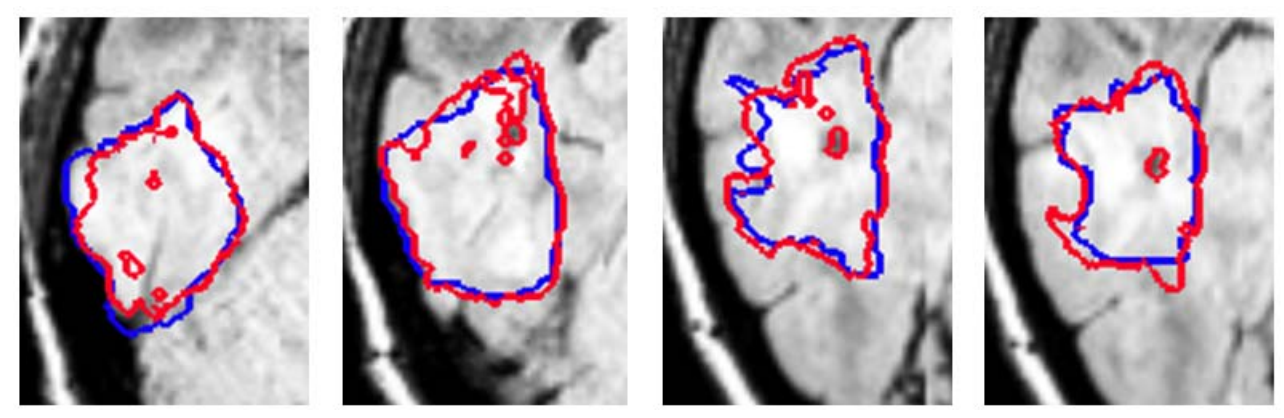

Figure 10: Four different axial slices of Patient B illustrating the prediction of the tumor evolution based on two different time points. Using the algorithm outcome at $t_{2}$, the tumor front is predicted at the next time point $t_{3}$. The red contour represent the predicted tumor boundary and the blue one represents the ground truth or the real tumor boundary.
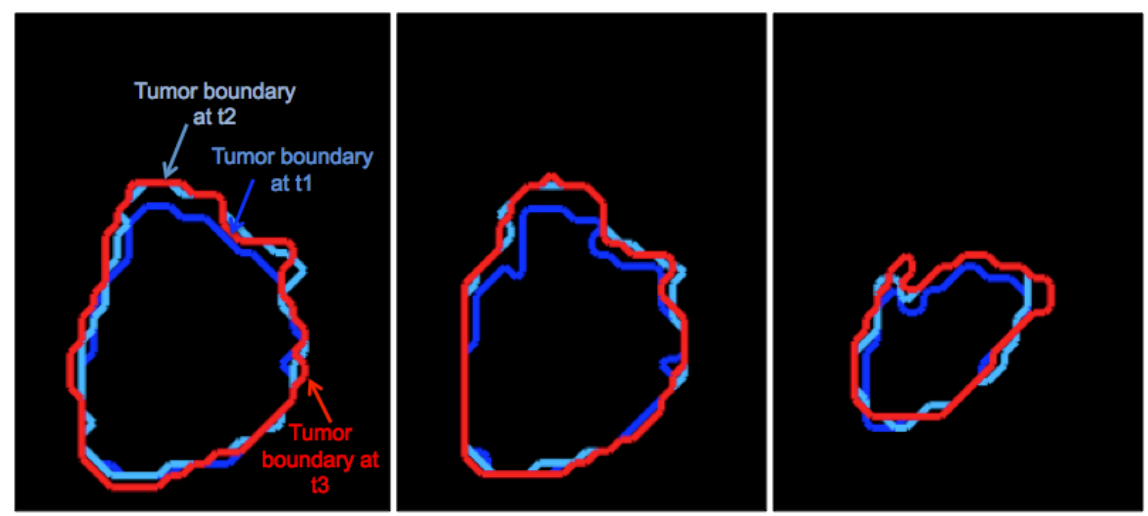

Figure 11: Patient C: three different axial slices of the manually segmented tumor boundary at $t_{1}$ in dark blue, at $t 2$ in dark blue and at $t_{3}$ in red. 